\title{
Authors' correction
}

\section{"Incidence of asthma and respiratory symptoms by sex, age and smoking in a community study". T.M.L. Eagan, P.S. Bakke, G.E. Eide, A. Gulsvik. Eur Respir J 2002; 19: 599-605.}

Unfortunately, one of the questions concerning respiratory symptoms was missing in the Appendix, as were the dates which separated the questions at baseline and follow-up. The authors would like to apologise for their mistake. The correct Appendix is reproduced below.

\section{Appendix}

The wording of the questions in 1985 and 1996/1997 regarding respiratory symptoms and asthma were identical, except for slight differences in the wording on wheezing and asthma:

1985:

Do you usually cough or clear your throat in the morning? (yes, no).

Do you usually cough during the rest of the day? (yes, no).

Do you usually have phlegm when coughing? (yes, no).

Do you have a cough for three months or more altogether during a year? (yes, no).

Are you more breathless than other people of your own age when walking uphill? (yes, no) (dyspnoea grade 1).

Are you breathless when you climb two flights of stairs at an ordinary pace? (yes, no) (dyspnoea grade 2).

Are you breathless walking at a normal pace on level ground? (yes, no) (dyspnoea grade 3 ).

Are you breathless while at rest? (yes, no) (dyspnoea grade 4).

Do you sometimes experience attacks of breathlessness? (yes, no).

Do you ever have wheezing in your chest (yes, no).

Have you ever been treated by a doctor or been hospitalized for asthma (yes, no, do not know).

1996/1997:

Have you ever had wheezing in your chest? (yes, no).

Have you ever been treated by a physician, or have you been hospitalized for asthma? (yes, no). 